\title{
Design and Dynamic Analysis of Drill Pipe Car Lifting Mechanism
}

\author{
Di Wu \\ Xi'an Research Institute,CCTEG,Xi'an710077,China \\ email:wudi@cctegxian.com
}

Key words: lifting mechanism; dynamic analysis; support reaction

\begin{abstract}
The paper designed a simple and neoteric lifting mechanism and calculated its dynamic analysis in order to meet the design requirements of the transferring vehicle in drill pipe car. And equivalent moment of inertia and equivalent of the torque are worked out by using MATLAB. Finally, it is a feasible mechanism showed by the computational results and provides a basis for the subsequent structural design and optimal design.
\end{abstract}

\section{Introduction}

The coal mine tunnel drilling rig is necessary equipment in the field of the gas extraction and the governance. With the progress of our country science and technology level, improving the level of coal mine tunnel drilling automation is imminent in order to meet the requirement of safe production of coal mine[1]. Designed an automatic lifting mechanism tunnel drilling rig is important for enhance the automation of tunnel drilling rig.

At present, the study is devoted to the engineering drilling machine of multi-functional automatic gear, and the problem that was partly solved. However, such devices are mostly large and complex structure and could not meet the need of drill automatically lifting rods in narrow downhole coal environment[2-3].

\section{Structure Design}

The paper designed a simple and neoteric lifting mechanism for coal mine tunnel drilling rig, including manipulator and drill pipe box. The role of manipulator is delivering the rods in drill pipe box to centerline of power head chuck and the automatic twist drill pipe was realized. The role of drill pipe box is stored the rods in accordance with the reservation number and arrangement. In order to realize the $t$ requirement of different level drilling process, the drill pipe box has elevator function itself.

Automatic lifting mechanism tunnel drilling rig was designed by the hydraulic cylinder driving fork scissors mechanism, having a large lifting force and the advantages of higher range. The fork scissors mechanism was link gear, the hinge pivot for horizontal movement was drive by the hydraulic cylinder, the drill pipe box was done exercise for vertical direction under the role of the linkage force transferring between the articulated points. The lowest position for drill pipe box movement was 0.3 meters, the highest position requirement was 0.7 meters. The four connecting rod length are same $L=0.8 \mathrm{~m}$. After calculating $\theta_{\text {high }}=61^{0}, \theta_{\text {low }}=22^{0}$. As the Fig.1 shows that the lifting mechanism is on drill pipe car. 


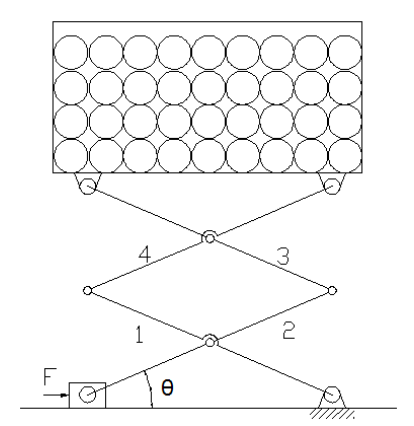

Fig.1 The lifting mechanism on drill pipe car

\section{Dynamic Analysis}

\section{Calculation of Equivalent Working Time}

In order to be easily calculated, the drill pipe box was the equivalent component only doing linear motion up and down in this design. The work that the equivalent force effects on equivalent component is equal to all the external forces acting on the system[4]. Based on the constant power, the equivalent force was:

$$
F_{e}=F \frac{v_{1}}{v}+G
$$

$F$--the force of hydraulic cylinder;

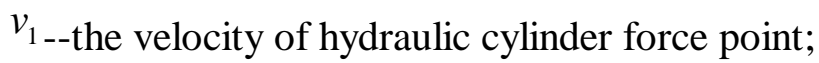

$v$-- the velocity of drill pipe box;

$G$-- the gravity of the tubing.

Planar mechanism for planar motion artifacts in its kinetic energy was:

$$
E_{k}=\frac{1}{2} m v_{s}^{2}+\frac{1}{2} j \omega^{2}
$$

$m_{\text {-- }}$ the mass of the component ;

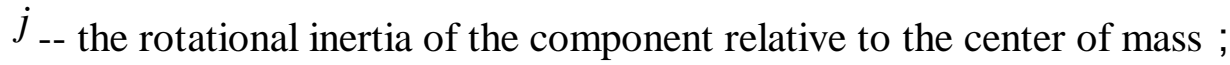

$v_{s_{--}}$the velocity of the center of mass ;

$\omega_{\text {-- }}$ the angular velocity of components.

Its kinetic energy for translational components only include the first item on the type, and the fixed-axis rotation components only include the second item on the type. According to the principle of equal convert the kinetic energy before and after, the equivalent mass was me.

$m_{e}=4 m_{1}\left(\frac{v_{s_{1}}}{v}\right)^{2}+4 J_{1}\left(\frac{\omega_{1}}{v}\right)^{2}+2 m_{2}\left(\frac{v_{s_{2}}}{v}\right)+m_{3}$

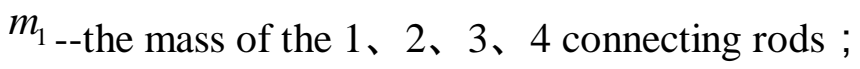

$v_{s_{1}}$-- the velocity of the center of mass of the $1,2 、 3,4$ connecting rods ;

$v_{--}$the velocity of equivalent component ;

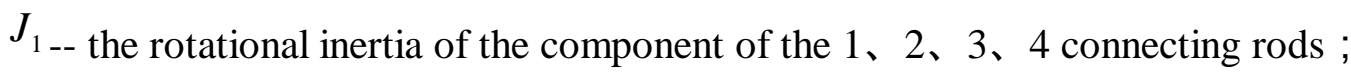

$\omega_{1--}$ the angular velocity of the $1,2 、 3,4$ connecting rods ;

$m_{2}$-- the mass of the slider ; 
$v_{s_{2}--}$ the slider center of mass velocity ;

$m_{3}$-- the mass of component 5 .

The transmission ratio is only associated with the location of institutions in the linkage of speed ratio transmission system. By a type (1), (2) the effectiveness and equivalent mass are only related to the transmission ratio, and has nothing to do with the real speed of mechanical drive components. The position of the institutions can be determined by the $\theta$ in this design as the Fig. 1 showed.

The type (1), (2) were rewrote into the following form according to the relationship between the calculated velocity ratio and $\theta$.

$$
\begin{aligned}
& F_{e}=F \frac{\sin \theta}{\cos \theta}-G \\
& m_{e}=4 m_{1}\left(\frac{1}{4 \cos \theta}\right)^{2}+4 J_{1}\left(\frac{1}{2 \cos \theta}\right)^{2}+2 m_{2}\left(\frac{\sin \theta}{\cos \theta}\right)^{2}+m_{3}
\end{aligned}
$$

As the Fig. 2 shows that four same slide rods, the first rod mass $\mathrm{m} 1=2.9311 \mathrm{e}+4 \mathrm{~g}$, $J_{1}=7.1567 e+9 m^{2} g$, the mass of top shelf was $m_{5}=6.7810 e+5 g$, the mass of the slider was $m_{6}=7.8205 e+5 g$, the gravity of tubing was $G=20000 \mathrm{~kg} \times 9.8 \mathrm{~m} / \mathrm{s}^{2}=196000 \mathrm{~N}$.

By the MATLAB programming, as the Fig. 2 shows that the variation law of equivalent mass and equivalent force with changing angle in rising up process. As the Fig. 3 shows that the variation law of equivalent mass and equivalent force with changing angle in falling down process.
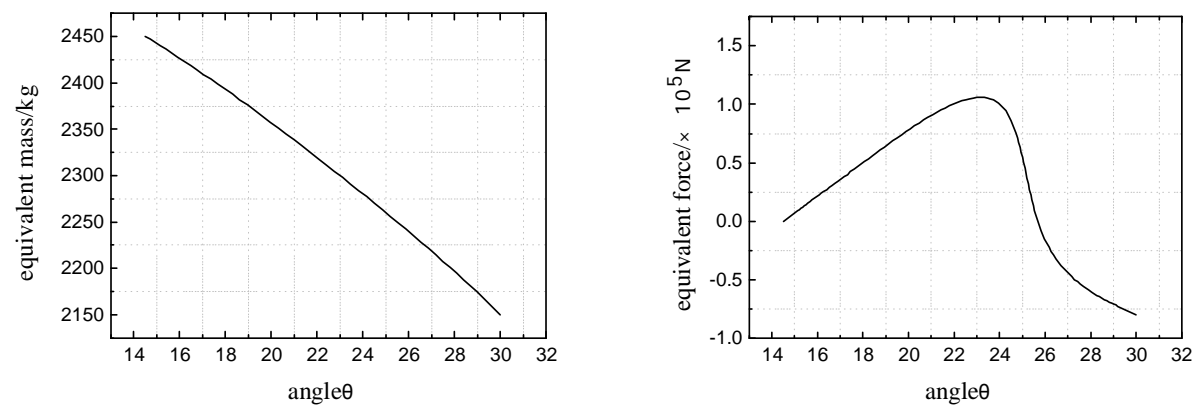

Fig.2 The variation law of equivalent mass and equivalent force with changing angle in rising up process
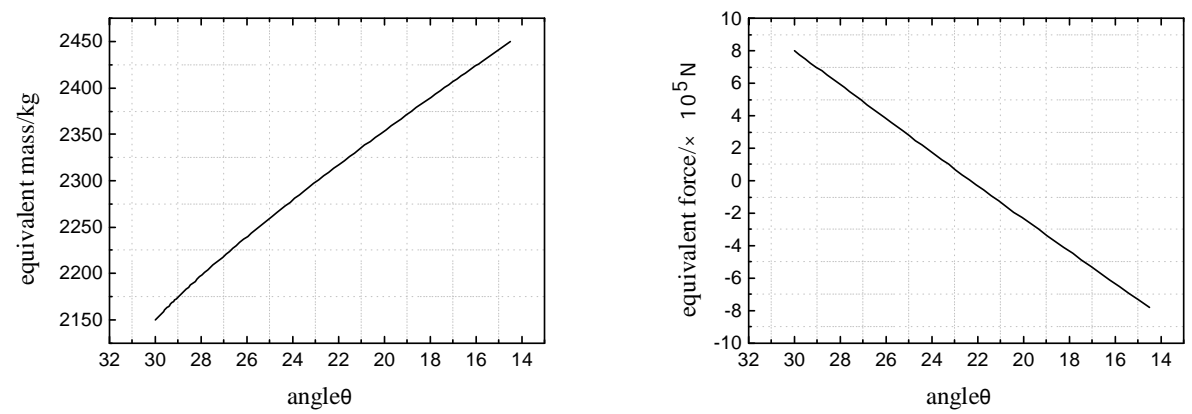

Fig.3 The variation law of equivalent mass and equivalent force with changing angle in falling down process

According to the kinetic energy theorem, the work was done by equivalent force is equal to the kinetic energy increment of equivalent components. Then

$\Delta E_{k}=W$ 
The velocity was changed from $v_{1}$ to $v_{2}$ when the component 5 was moved from $\theta 1$ to $\theta$ 2.Then the type was:

$$
\frac{1}{2} m_{e} v_{2}^{2}-\frac{1}{2} m_{e} v_{1}^{2}=\int_{\theta_{1}}^{\theta_{2}} 2 L F_{e} \cos \theta d \theta
$$

In the rising up process, the lowest position was taken for the initial state, $v_{1}=0 \mathrm{~m} / \mathrm{s}$, $\theta_{1}=14.5^{\circ}$.By the type (3)、 ( 4 )、(6):

$$
v_{u}=\sqrt{\frac{(\sqrt{15} / 4)-\cos \theta F+(1 / 4-\sin \theta) G}{m_{e} / 4}}
$$

In the falling down process, the highest position was taken for the initial state, , $v_{1}=0 \mathrm{~m} / \mathrm{s}$, $\theta_{1}=30^{\circ}$. By the type (3)、( 4 )、(6):

$$
v_{d}=\sqrt{\frac{(\cos \theta-\sqrt{3} / 2) F+(\sin \theta-1 / 2) G}{m_{e} / 4}}
$$

By the MATLAB programming, the figure 1.4 shows that the velocity law of equivalent component 5 in the rising up and falling down process.
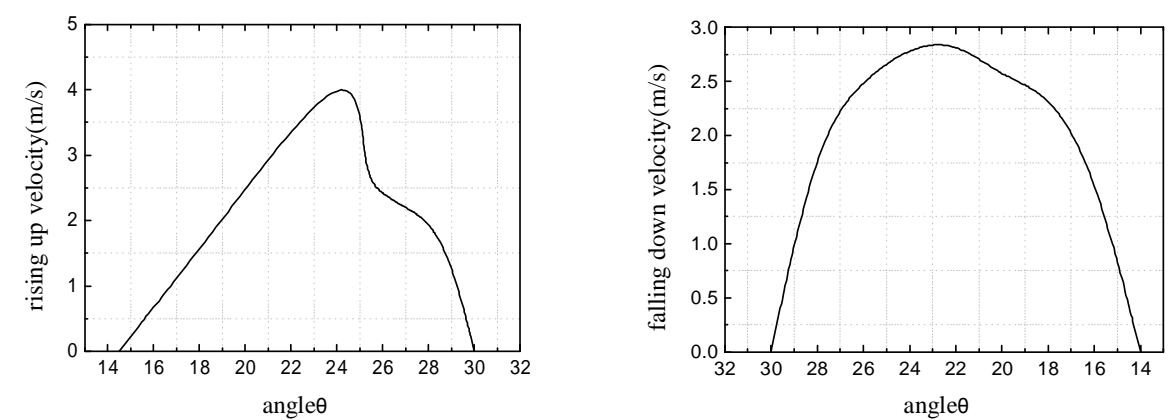

Fig.4 The velocity law of equivalent component 5 in the rising up and falling down process

If the equivalent component 5 rose up dy at the vertical direction, the function is $d t=\frac{2 L \cos \theta}{2(\theta)}$, the time integral equation is $\int_{\theta_{1}}^{\theta_{2}} \frac{2 l \cos }{v_{u}(\theta)} d \theta$. By the MATLAB programming, the rising-up time was $0.6379 \mathrm{~s}$, and the falling-down time was $0.519 \mathrm{~s}$, the total response time was $1.1569 \mathrm{~s}$.

\section{The Support Reaction Calculation of Component}

Because the connecting rod 2, 3 and 1, 4 are symmetry, according to the structure symmetry, the support reaction of the connecting rod 2, 3 and 1, 4 are also symmetry, and only the connecting rod 2, 3 support reaction was calculated[5].

As the Fig.5 shows that the simplified force diagram of component. 


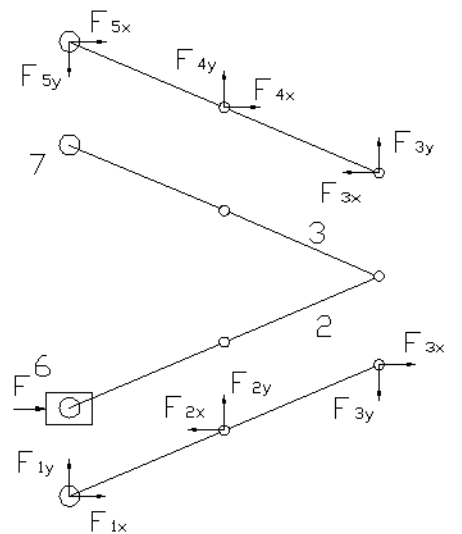

Fig.5 The simplified force diagram of component

The force and moment balance equations of component 2 was:

$$
\left\{\begin{array}{l}
F_{1 x}-F_{2 x}+F_{3 x}=m_{2} \alpha_{2} x \\
F_{1 y}+F_{2 y}-F_{3 y}=m_{2} \alpha_{2} y \\
q_{2 y} F_{1 x}-q_{2 x} F_{1 y}-p_{2 y} F_{3 x}-p_{2 x} F_{3 y}=J_{2} \theta_{2}
\end{array}\right.
$$

The force and moment balance equations of component 3 was:

$$
\left\{\begin{array}{l}
F_{5 x}-F_{4 x}+F_{3 x}=m_{3} \alpha_{3} x \\
F_{4 y}+F_{3 y}-F_{5 y}=m_{3} \alpha_{3} y \\
p_{3 x} F_{3 y}-p_{3 y} F_{3 x}+q_{3 x} F_{5 y}-q_{3 y} F_{5 x}=J_{3} \theta_{3}
\end{array}\right.
$$

The force and moment balance equations of component 6 was:

$$
\left\{\begin{array}{l}
-F_{1 x}=m_{6} \alpha_{6 x}-F \\
F_{1 y}-F_{u}=0
\end{array}\right.
$$

The force and moment balance equations of component 7 was:

$$
\left\{\begin{array}{l}
F_{5 x}-f=m_{7} \alpha_{7 x} \\
F_{5 y}=G
\end{array}\right.
$$

By the MATLAB programming and using linear equation, the support reaction of the $1 、 2 、 3 、 4$ 、 5 point in the Fig. 5 was:

$$
\begin{array}{lllllllllll}
F_{1 x} & F_{1 y} & F_{2 x} & F_{2 y} & F_{3 x} & F_{3 y} & F_{4 x} & F_{4 y} & F_{5 x} & F_{5 y}
\end{array}
$$

The maximum force at the horizontal and vertical direction of the $1,2,3,4,5$ point respectively was: (630,146)、( 91,272)、( 306,136 )、( 212,240 ) and ( 133,190) (unit: KN).

\section{Conclusion}

The dynamics of fork scissors delivering pipes lifting mechanism was analyzed, the total response time was found out for the mechanism motion at various points in the process of reaction. As the force variation law and velocity law shows when the angle was $25^{\circ}$,the velocity was suddenly changed because the velocity of the mechanism motion to the highest position was zero, the hydraulic cylinder thrust force must be variable force. By the MATLAB programming, it is a feasible mechanism showed by the computational results and provides a basis for the subsequent structural design and optimal design. 


\section{References}

[1] PanJiao Li. Two Hinged Fork Scissors Mechanism Structure Design and Application of Hydraulic Lifting Platform. Mechanical Engineer. 2015 ( 6 ) : 85-88.

[2] SiHua Chen; Guo Zhou; ZhongKai Deng, The Design of the Shear Fork Hydraulic Lift. Mechanical and Electrical Equipment. 2015 ( 32 ) : 34-36.

[3] XueLong Zhao; Qiang Wen; QiaoZhen Liu, Stability Calculation of Shear Fork Aerial Work Platform. 2015 ( 7 ) : 87-90.

[4] Ce Zhang, Mechanical Dynamics[M].BeiJing: Higher Education Press, 2000:36-48.

[5] Zi Lu; LiHua Wang; YuSong He; The Optimization of Shear Fork Type Hydraulic Lifting Platform. New Technology \& New Process , 2013 ( 11 ) : 11-15. 